\title{
Bilateral Tension Band Osteosynthesis in Fixation of Intracapsular Supracondylar Distal Humeral Fractures
}

\author{
Amro I Zanfaly, MD*, Mohammed S Elattar, MD, Mohammed Elsadek, MD \\ Orthopedic Surgery Dept. Zagazig University, Egypt \\ yousufmmkh@gmail.com
}

Abstract

Background: The distal humeral fractures in adults are complex and technically demanding. Most cases often need operative treatment. Many methods of fixation were evolved with the better imaging and new techniques and implants.

Patients and Methods: Fourteen patients, nine males and five females, with fracture of the distal humerus were treated with double tension band wiring through the period from May 2014 to June 2018. Patient's age ranged from 26 - 52 (average: 36 y). DASH score (The Disabilities of the Arm, Shoulder and Hand) [12] was used for subjective evaluation; and pain at last follow-up was measured using an analog scale ( 0 being no pain and 10 significant pain).

Results: Clinically: By the end of follow up the DASH score ranged from 5 to 21 (average: 13.8 points). The pain scale analog ranged from 0 to 3 with average of 1.2 points. Radiologically: All fractures were united with average of 16 weeks range (12-18 weeks). There was no elbow stiffness or heterotopic bone ossification.

Conclusion: trans-osseous tension band wiring technique is an acceptable technique for treatment of supracondylar intracapsular fractures with good results. As regarding the small number of patients of this study, we recommend this technique for further research and large number of patients.

\section{INTRODUCTION}

Although rare, the distal humeral fractures in adults are complex and technically demanding in management [1, 2]. Most cases often need operative treatment. Many methods of fixation were evolved with the better imaging and new techniques and implants. Using single screws, Steinmann pins or multiple K-wires in type C distal humerus fractures is associated with poor results $[3,4]$. In recent reviews dual plate fixation with strong plate on each column is indicated for all adult fractures involving both columns of the distal part of the humerus [5, 6]. One author even states that failure to adhere to the principles of rigid fixation with a strong plate on each column can dramatically increase nonunion rates [3]. Previous reports in the literature reported good results using double tension band osteosynthesis or combining tension bands with other fixation methods in supra and intercondylar humeral fractures instead of double plating for fixation of the condylar block to the shaft [7-9].

The intercondylar area being intra articular completely provides very little space for the use of various rigid implants. With the use of anatomical plates, more than one screw can be taken in the intercondylar region, but without purchase on the opposite cortex which makes fixation not rigid [2]. A simple useful method was devised for rigidly fixing the supracondylar fractures region and maintaining as normal as possible the anatomy approximatingthe cortices fixation. This method converts a very unstable fracture of humerus into a stable reconstruct even in osteoporosis and open cases and allow early mobilization [10].

In this technique the principle of tension band wiring hasbeen utilized over screws + /- K-wires by trans osseous route with reduction of intra articular anatomy if present. The aim of this study was to evaluate the objective 
Bilateral Tension Band Osteosynthesis in Fixation of Intracapsular Supracondylar Distal Humeral Fractures

and subjective results achieved afterBilateral Tension Band Osteosynthesis in Fixation of Intracapsular Supracondylar Distal Humeral Fractures in14 adults.

\section{Patients ANd Methods}

\section{Patients}

Fourteen patients, nine males and five females, with fracture of the distal humerus were treated with double tension band wiring through the period from May 2014 to June 2018. Patient's age ranged from $26-52$ (average: 36 y). Plain radiography (antero-posterior and lateral views) were done for all patients. Ten fractures were type A2 and four type A3 according to (AO/OTA) classification [11], and fourcases were open fractures. All lesions were supracondylar intracapsular with transverse orientation compromised both columns of the distal humerus. In four cases the fracture had intercondylar element passing through the olecranon fossa (Figs. 1). Two patients had ulnar nerve injury before operation.

\section{Surgical Technique}

All patients were selected randomly the only criteria was a supracondylar fracture of lower end of humerus with intercondylar extension in four cases only. Intercondylar portion of fracture was first reduced and held with AO reduction clamp as anatomical as possible. Then cancellous screw was passed from medial to lateral aspect crossing intraarticular fracture line perpendicularly in four cases (Fig. 1).
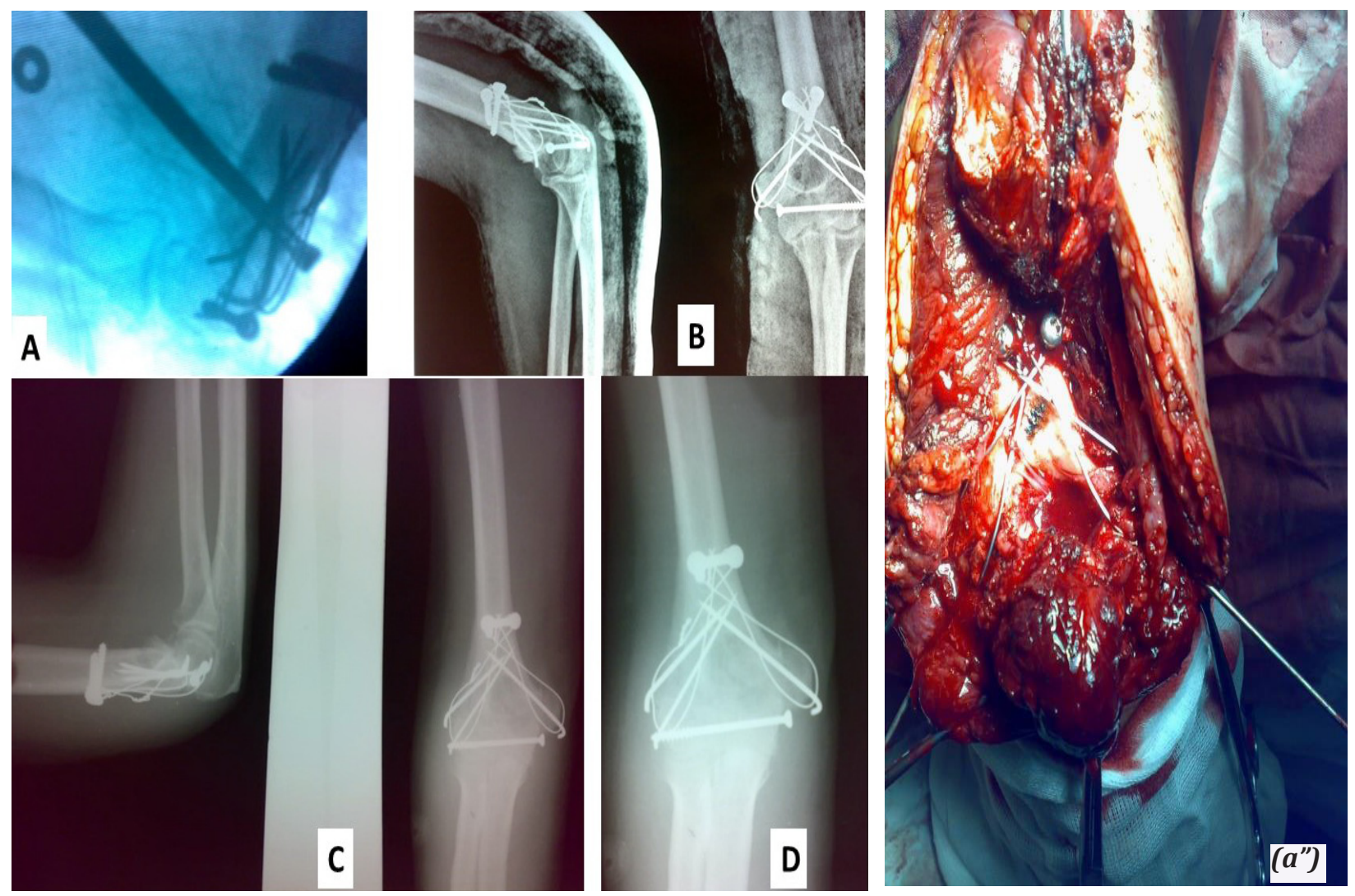

Figure1. intraoperative fluoroscopy assessment of reduction (a) and an intraopwerative photo showing posterior triceps tongue elevating approach $\left(a^{\prime}\right)$ lateral and antero-posterior $x$ ray view immediate postoperative and application of above elbow splint (b) 6 weeks postoperative views (c) 16 weeks follow up x ray (d) 
All patients were operated on in lateral decubitus under regional anesthesia in nine cases, and general anesthesia in five cases. The arm was hanged over a radiolucent support, with the elbow free for $90^{\circ}$ of flexion. A posterior approach (Alonso - Llames) was used in eight cases and posterior triceps tongue elevating approach in six cases $[3,4,6,8,9$ and 14]. After exploration of ulnar nerve, in five cases a 1.8- or 2-mm K-wires were used; they were placed entering through the epicondyles to the fracture line, then thy wires were used as joysticks to achieve reduction of the fragments. Once reduction was accepted, the K-wires were placed to the medial and lateral cortices of the proximal fragment, after that, a hole with a $2.7-\mathrm{mm}$ drill was performed at the proximal fragment on each side, and a 1.6- or 2-mm stainless-steel wire was driven through it, then crossed to make a figure of eight, and passed through the distal soft-tissues, distally to the entry point of the K-wires. Once both tension bands were in place they were adjusted simultaneously to achieve a compression as symmetrical as possible. Once the reconstruction was tensed elbow range of motion was checked to assess construct stability under fluoroscopy.In other nine cases, the stainless steel wire was passed through trans osseous canal fixed under the heads of two screws proximally to make two tension bands one lateral and one medial.. Distally, the wire passed through the soft tissue under two crossing k-wires distally into the lower condylar region and back again in the proximal part. Now tension was applied by twisting the free ends of the stainless steel wire on the lateral and medial side (Fig. 2). While tensioning, compression was achieved at the fracture site and also the stability was confirmed on fluoroscopy. This method gave us four cortices fixation in the lower supracondylar region. In cases of open fractures the trans osseous canal we used only k-wires instead of screws to be minimal implants to safe guard against infection of implants.
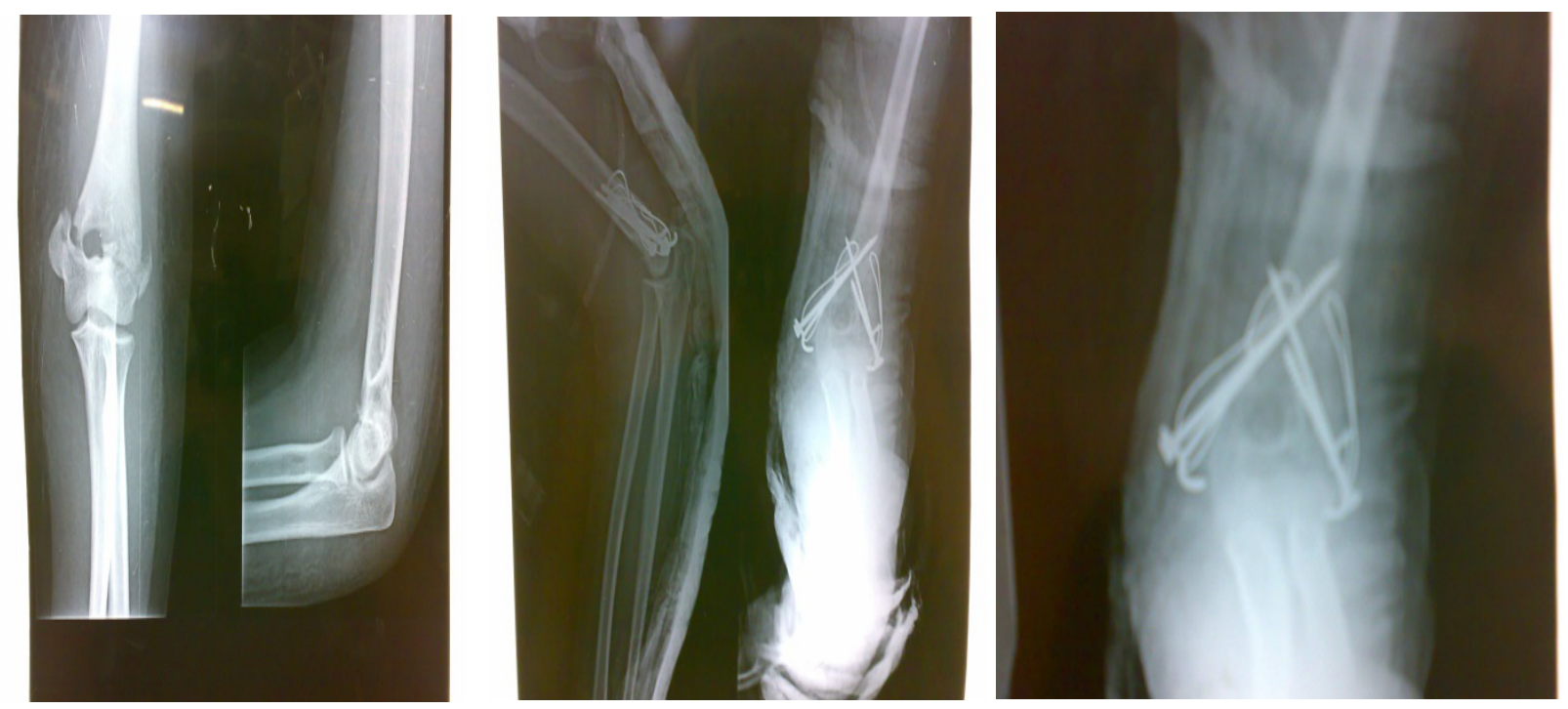

Figure2. preoperative x ray showing supracondylar fracture with its transverse orientation (a) lateral and anteroposteriorx ray view immediate postoperative and application of above elbow splint (b) 12 weeks postoperative views (c)

\section{Postoperative Management}

Postoperatively, the arms were immobilized in posterior splint for six weeks, then controlled active and passive elbow joint motion was started. Weekly visits in the first month, followed by monthly visits for 6 months were advised to the patients. Follow up included plain x-ray using antero-posterior and lateral X-rays for union judgment. Objective evaluation of range of motion was performed using a goniometer. DASH score (The Disabilities of the Arm, Shoulder and Hand) [12] was used for subjective evaluation; and pain at last follow-up was measured using an analog scale (0 being no pain and 10 significant pain). 


\section{RESULTS}

\section{Clinically}

By the end of follow up the DASH score ranged from 5 to 21 (average: 13.8 points). The pain scale analog ranged from 0 to 3 with average of 1.2 points. The range of motion of the elbow at the last follow-up ranged from $95-125^{\circ}$ (average: $105^{\circ}$ ). The loss of the elbow extension ranged from $15-25^{\circ}$ (average: $16.5^{\circ}$ ). The range of pronation and supination was complete, except in two patients who had lost $25^{\circ}$ of supination.

\section{Radiologically}

All fractures were united with average of 16 weeks range (12-18 weeks) (Fig. 3). The two patients with preoperative ulnar nerve symptoms recovered completely within four months postoperative. Three cases with superficial wound infectionswhich were relieved after extraction of k-wires 45 days postoperative and repeated dressing. There was no elbow stiffness or heterotopic bone ossification.


Figure3. preoperativeX ray (a) Immediate postoperative x ray and application of above elbow splint (b)12 weeks follow up $x$ ray showing fracture union (c)

\section{DISCUSSION}

The fractures of the distal humerus in adults is a challenge for orthopedic surgeons due to the complex anatomy of the elbow joint [1]. The highly constrained elbow joint nature, narrow supracondylar isthmus, and articular comminution especially within the olecranon fossa are the main difficulties in reconstruction [4]. The principles for distal humeral fractures management are anatomical reduction, rigid fixation to allow early mobilization. The double plating fixation was recommended by (AO) in 1960S for rigid fixation and early mobilization [3, 5, 7,9 , and 12]. In the method described in this work, compression, and stable fixation could be achieved by trans osseous tension band wiring for the fixation of intracapsular supracondylar fractures. Tension band principal here were applied by the common extensor origin at lateral condyle and common flexor origin at medial condyle as contraction of both muscle groups turned the distraction forces into compression forces.

Although the percutaneous K-wires can be used in the fixation of supracondylar fractures of distal humerus in children, their use in adults has bad results and not recommended [2, 4]. On the other hand, some authors 
Bilateral Tension Band Osteosynthesis in Fixation of Intracapsular Supracondylar Distal Humeral Fractures

reported the use of tension band wiring in treating supracondylar fractures of the distal humerus with good results [7 - 9].

Zhao et al. [7] treated 24 patients with comminuted intra-articular fractures with double band osteosynthesis and reported $83 \%$ excellent or good results.

Comparable results were reported by Houben et al. [8] in ten patients with type $\mathrm{C}$ distal humerus fractures, five of them treated with double tension band osteosynthesis and five with double plate, for fixation of the condylar block to the shaft of the humerus. Allende et al. [9] reported on the use of tension bands in combination with other fixation methods for the treatment of intercondylar distal humerus fractures, with conclusion that it can be good alternative method in the treatment of comminuted distal humeral fractures with osteoporotic bone. In this work, the average age was 36 years, all fractures were united, and postoperative motion in all cases was allowed as the early motion was one of the main objectives in this study. During fixation four cortices contact could be achieved and this stability gave the ability to mobilize the patient early as the tension bend wiring technique is a dynamic mode of fixation.

The locked plates have not yet proved to be superior to other fixation methods for distal humerus fractures, and their use remains controversial. Lower profile plates and smaller screws are showing some results.

This method of trans osseous intercondylar tension band wiring can be useful technique in such situations that allow the surgeon to maintain the original articular congruity needed to prevent posttraumatic arthritis, which allows for faster and progressive postoperative rehabilitation. On the other hand, some drawbacks in the use of locked plate as it needs more extensive soft-tissue dissection, expensive, more time consuming, and technically more demanding. Also, the locked plates do not always match the anatomy of the reconstructed distal humerus and cannot be molded to adapt to the reconstruction achieved. The tension band fixation method adapts to the modern principles of fracture fixation established by the AO (flexible and biological fixation, that will allow prompt motion) as it requires less periosteal stripping and muscle, technically less demanding, faster and cheaper than plate fixation.

\section{CONCLUSION}

Transosseous tension band wiring technique is an acceptable technique for treatment of supracondylar intracapsular fractures with good results.As regarding the small number of patients of this study, we recommend this technique for further research and large number of patients.

\section{REFERENCES}

1. Papaioannou N, Babis GC, Kalavritinos J, Pantazopoulos T (1995) Operative treatment of type C intraarticular fractures of the distal humerus: the role of stability achieved at surgery on final outcome. Injury 26:169-173.

2. Ulusal AE, Boz U, Sertoz Z, Ustaoglu RG (2006) Approaches to distal humerus fractures in adults and comparison of treatment results. Acta Orthop Traumatol Turc 40:22-28.

3. Nauth A, McKee MD, Ristevski B, Hall J, Schemitsch (2011) Distal humeral fractures in adults. J Bone Joint Surg 93-A: 686-700.

4. Evans EM. Supracondylar-Y fractures of the humerus. J Bone Joint Surg Br. 1953; 35-B (3):371-5.

5. Sanchez-Sotello J (2012): Distal humerus fractures: role of internal fixation and elbow arthroplasty. J Bone Joint Surg 94-A:556-568 
Bilateral Tension Band Osteosynthesis in Fixation of Intracapsular Supracondylar Distal Humeral Fractures

6. Jupiter JB. The management of nonunion and malunion of the distal humerus--a 30-year experience. J Orthop Trauma. 2008; 22(10):742-50.

7. Zhao J, Wang X, Zhang Q (2000): Surgical treatment of commnuted intra-articular fractures of the distal humerus with double tension band osteosynthesis. Orthopedics 23:449-452.

8. Houben PFJ, Bongers KJ, Wildenberg FAJM (1994) Double band osteosynthesis in supra- and trans condylar humeral fractures. Injury 25:305-309.

9. Allende CA, Allende BT, Allende BL, Bitar I, Gonzalez G (2004) Intercondylar distal humerus fracturessurgical treatment and results. Chir Main 23:85-95.

10. Schemitsch EH, Tencer AF, Henley MB. Biomechanical evaluation of methods of internal fixation of the distal humerus. J Orthop Trauma. 1994; 8(6):468-75.

11. Hudak PL, Amadio PC, Bombardier C. Development of an upper extremity outcome measure: the DASH (disabilities of the arm, shoulder and hand). The Upper Extremity Collaborative Group (UECG); Am J Ind Med. 1996 Jun; 29(6):602-8.

12. Allende C, Allende BT (2009) Post-traumatic distal humerus non-union. Int Orthop 33:1289-1294

Citation: Amro I Zanfaly, Mohammed S Elattar, Mohammed Elsadek. "Bilateral Tension Band Osteosynthesis in Fixation of Intracapsular Supracondylar Distal Humeral Fractures". American Research Journal of Orthopedics and Traumatology. 2018; 3(1): 1-6.

Copyright (c) 2018 Amro I Zanfaly, Mohammed S Elattar, Mohammed Elsadek. This is an open access article distributed under the Creative Commons Attribution License, which permits unrestricted use, distribution, and reproduction in any medium, provided the original work is properly cited. 